\title{
TWENTY-FOUR GENES ARE UPREGULATED IN PATIENTS WITH HYPOSPADIAS
}

\author{
Karabulut R ${ }^{1}$, Turkyilmaz Z1 , Sonmez K ${ }^{1}$, Kumas $\mathrm{G}^{2}$, Ergun $\mathrm{SG}^{2}$, Ergun $\mathrm{MA}^{2, *}$, Basaklar AC ${ }^{1}$
}

*Corresponding Author: Mehmet A. Ergun, M.D., Ph.D., Department of Medical Genetics, Gazi University Faculty of Medicine, Besevler, Ankara, Turkey; Tel.: +90 312 2024637; Fax. +90 312 2024635; E-mail: aliergun@gazi.edu.tr

\section{ABSTRACT}

Hypospadias is a congenital hypoplasia of the penis, with displacement of the urethral opening along the ventral surface, and has been reported to be one of the most common congenital anomalies, occurring in approximately 1:250 to 1:300 live births. As hypospadias is reported to be an easily diagnosed malformation at the crossroads of genetics and environment, it is important to study the genetic component in order to elucidate its etiology. In this study, the gene expression profiles both in human hypospadias tissues and normal penile tissues were studied by Human Gene Expression Array. Twentyfour genes were found to be upregulated. Among these, ATF3 and CYR61 have been reported previously. Other genes that have not been previously reported were also found to be upregulated: $B T G 2$, CD69, CD9, DUSP 1, EGR1, EIF4A1, FOS, FOSB, HBEGF, HNRNPUL1, IER2, JUN, JUNB, KLF2, NR4A1, NR4A2, PTGS2, RGS1, RTN4, SLC25A25, $S O C S 3$ and ZFP36 $(p<0.05)$. Further studies including genome-wide association studies (GWAS) with expression studies in a large patient group will help us for identifiying the candidate gene(s) in the etiology of hypospadias.

Keywords: Hypospadias; Gene expression; Microrarray.

\footnotetext{
${ }^{1}$ Department of Pediatric Surgery, Gazi University Faculty of Medicine, Ankara, Turkey

${ }^{2}$ Department of Medical Genetics, Gazi University Faculty of Medicine, Ankara, Turkey
}

\section{INTRODUCTION}

Hypospadias is a congenital hypoplasia of the penis, with displacement of the urethral opening along the ventral surface, often associated with dorsal hooded foreskin and chordee [1]. The anatomical location of the misplaced urethral meatus determines the severity of this anomaly with the severity increasing from distal to proximal [2]. Hypospadias has also been reported to be one of the most common congenital anomalies, occurring in approximately $1: 250$ to $1: 300$ live births [3].

Although, the etiology of hypospadias remains unknown, a genetic component in the transmission of this birth defect has been suggested so it seems to be multifactorial [4]. In addition, $30.0 \%$ of severe hypospadias can be attributed to defects in the synthesis of testosterone or adrenal steroid hormones, receptor defects, syndrome-associated hypospadias, chromosomal anomalies, and/or defects in other genetic factors [2]. Thus, hypospadias may be a highly heterogeneous condition subject to multiple genetic and environmental factors [5].

With regard to molecular biology and microarray technology, it appears that hypospadias is potentially related to disrupted gene expression [6]. Previous studies revealed candidate genes including $W T 1$, SF1, BMP4, BMP7, HOXA4, HOXB6, AR, FGF8, FGFR2, HSD3B2, SRD5A2, ATF3, MAMLD1, MID1, BNC2, ESR1, ESR2, ATF3, DGKK, CYP1A1, GSTM1, GSTT1, CTGF, CYR61 and EGF [1]. Also, in order to study the epigenetic modification 


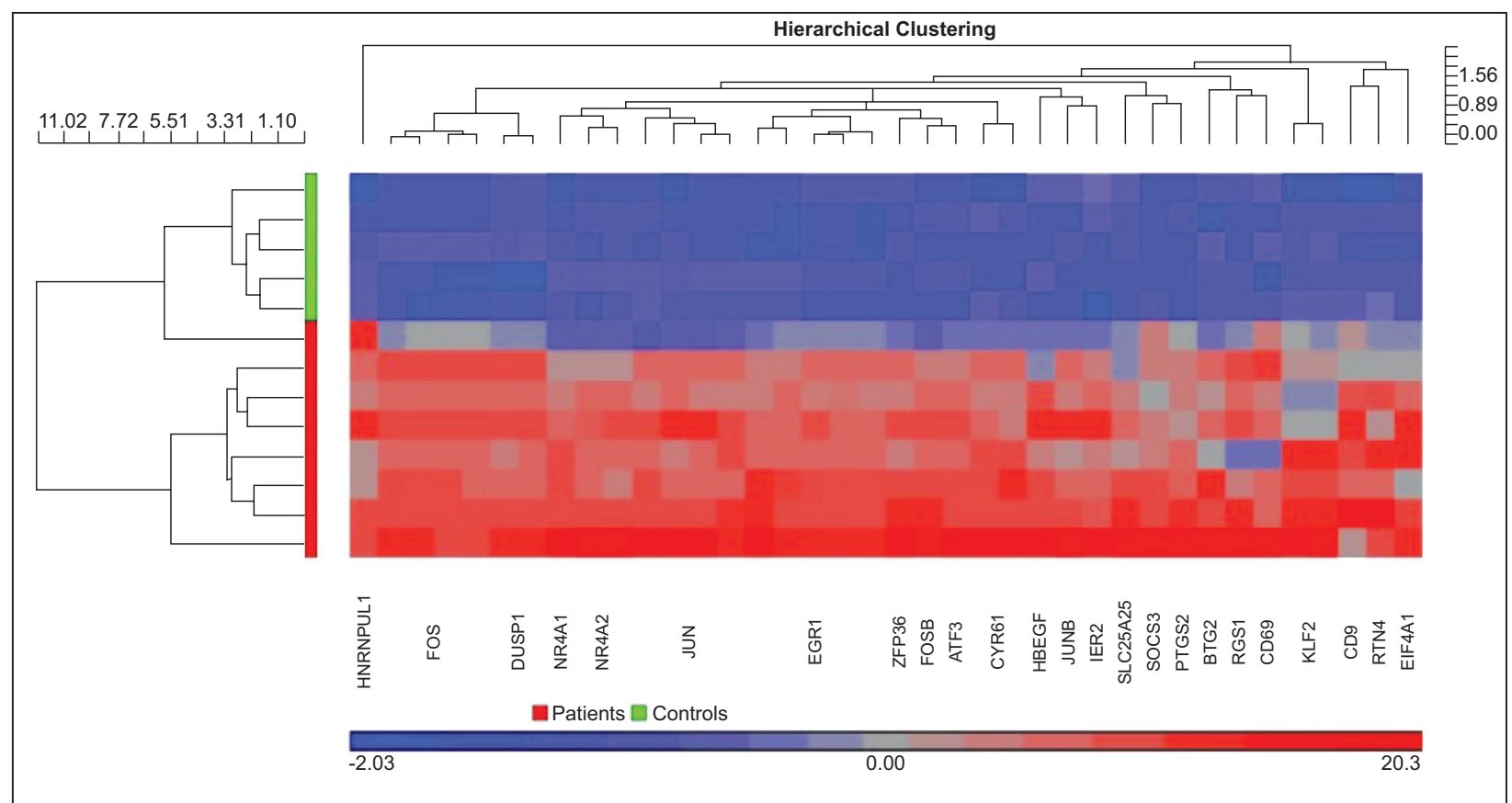

Figure 1. Gene tree of the top 24 upregulated genes whose expression significantly changed greater than 2-fold between hypospadias and control groups.

of DNA methylation in hypospadias, genome-wide DNA methylation profiling was performed, and the $S C A R B 1$ and $M Y B P H$ genes have been reported to be involved in the etiology of hypospadias [7].

As hypospadias is reported to be an easily diagnosed malformation at the crossroads of genetics and environment, it is important to study the genetic component in order to elucidate its etiology [5]. Thus, in this study, our aim was to study the gene expression profiles both in human hypospadias tissues compared with that in normal penile tissues.

\section{MATERIALS AND METHODS}

Patients. A total of eight patients with isolated distal (subcoronal) hypospadias (mean age 6.8; range 2-10 years) and five healthy circumcised controls (mean age 6.5; range 2-10 years) were enrolled in this study. The penile skin tissue specimens obtained at surgery during hypospadias repair or elective circumcision were divided into two groups: children with hypospadias $(n=8)$, and normal controls $(n=5)$. Informed consent was obtained from the parents/guardians of all the children.

Samples were flash frozen with liquid nitrogen and stored at $-80{ }^{\circ} \mathrm{C}$ until further processing. The gene expression studies were assessed using
Genechip ${ }^{\circledR}$ Primeview ${ }^{\mathrm{TM}}$ Human Gene Expression Array (Affymetrix, Santa Clara, CA, USA) which contain more than 53,000 probes including over 36,000 transcripts and variants.

RNA Isolation. Samples were distrupted and powdered under liquid nitrogen with pestle and mortar. Tissues were transferred into microcentrifuge tubes and lysated with $1 \mathrm{~mL}$ of TRIzol Reagent (Invitrogen, Carlsbad, CA, USA). All samples were homogenized using Qiashredder (Qiagen, Valencia, CA, USA); $0.2 \mathrm{~mL}$ chloroform was added to homogenized samples and centrifuged at $12,000 \mathrm{~g}$ for 15 min. Upper aqueous phase was transferred into a new microcentrifuge tube and isolation of the highpurity total RNA was perfomed using the RNeasy ${ }^{\circledR}$ Mini Kit (Qiagen) following the manufacturer's specifications. The quantity and purity of RNA was determined using the NanoDrop 1000 spectrophotometer (Thermo Scientific, Wilmington, DE, USA) at $260 \mathrm{~nm}$.

Gene Expression Studies. Genechip ${ }^{\circledR}$ Primeview $^{\mathrm{TM}}$ Human Gene Expression Array (Affymetrix) was used for gene expression studies. Five hundred ng of total RNA was reverse transcribed, amplified and biotin-labelled with Genechip 3'IVT (in vitro transcription) Express Kit (Affy-metrix) according to manufacturer's instructions. aRNAs were then purified with magnetic beads and after fragmenta- 
Table 1. Significantly expressed genes that changed more than 2-fold between hypospadias and control groups.

\begin{tabular}{|c|c|c|c|}
\hline Gene & Gene Title & Function & $\begin{array}{c}\text { Fold- } \\
\text { Change }\end{array}$ \\
\hline FOS & FBJ murine osteosarcoma viral oncogene homolog & apoptosis, transcription & 16.649 \\
\hline FOSB & FBJ murine osteosarcoma viral oncogene homology B & transcription & 14.9028 \\
\hline ATF3 & Activating transcription factor 3 & transcription & 13.2691 \\
\hline NR4A1 & Nuclear receptor subfamily 4 , group A, member 1 & apoptosis, signaling & 10.5824 \\
\hline PTGS2 & Prostaglandin-endoperoxide synthase 2 & metabolism & 9.44542 \\
\hline DUSP1 & Dual specificity phospatase 1 & signaling & 7.95108 \\
\hline SOCS3 & Suppressor of cytosine signaling 3 & signaling & 7.24912 \\
\hline CD69 & CD69 molecule & receptor activity & 7.06541 \\
\hline CYR61 & Cysteine-rich, angiogenic inducer 61 & signaling & 6.99616 \\
\hline$J U N$ & Jun proto-oncogene & transcription & 6.4233 \\
\hline EGR1 & Early growth response 1 & transcription & 6.37844 \\
\hline NR4A2 & Nuclear receptor subfamily 4 , group A, member 2 & signaling & 5.27591 \\
\hline$R G S 1$ & Regulator of G-protein signaling 1 & signaling & 4.25487 \\
\hline$H B E G F$ & Heparing-bindingEGF-like growth factor & signaling & 3.61757 \\
\hline$J U N B$ & Jun B proto-oncogene & transcription & 3.57188 \\
\hline$C D 9$ & CD9 molecule & signaling & 3.37889 \\
\hline RTN4 & Reticulon 4 & protein binding & 3.21246 \\
\hline IER2 & Immediate early response 2 & transcription & 3.05546 \\
\hline$S L C 25 A 25$ & $\begin{array}{l}\text { Solute carrier family } 25 \\
\text { (mitochondrial carrier; phosphate carrier), member } 25\end{array}$ & transporter & 2.92541 \\
\hline ZFP36 & Zinc finger protein 36, C3H type, homology (mouse) & transcription & 2.61352 \\
\hline$K L F 2$ & Kruppel-like factor 2 (lung) & transcription & 2.39853 \\
\hline EIF4A1 & Eukaryotic translation initiation factor $4 \mathrm{~A} 1$ & translation & 2.36873 \\
\hline$B T G 2$ & BTG family, member 2 & transcription & 2.33987 \\
\hline HNRNPUL1 & Heterogeneous nuclear ribonucleoprotein U-like 1 & transcription & 2.0158 \\
\hline
\end{tabular}

tion of purified biotinylated aRNAs, samples were loaded to Genechips for subsequent hybridization. Afterwards, Genechips were washed and stained on the Fluidics station with specified protocol.

Statistical Analysis. Signal intensities were acquired by Genechip Scanner 3000 7G (Affymetrix) to generate cell intensity files (CEL). Statistical analysis was performed using Partek Genomics Suite software (Partek Inc., St. Louis, MO, USA). Robust multi-array average (RMA) algorithm was used for data normalization. One-way analysis of variance (ANOVA) was used when $>2$ groups were compared, followed the by $t$-test. The statistical significance level was set at false discovery rate ((FDR) $p<0.05$ to minimize false identificaion of genes. Greater than 2-fold changes were analyzed for up or down regulated genes. Hierarchical clustering based on genes and samples was performed with Partek Genomics Suite Software (Partek Inc.).

\section{RESULTS}

Gene Microarray Analyses. A total of 24 genes were found to be upregulated (Figure 1). The upregulation of the ATF3 and CYR61 genes that were previosuly reported have been detected in hypospadias patients $(p<0.05)$. Other genes that were not previously reported, were also found to be upregulated: BTG2, CD69, CD9, DUSP1, EGR1, EIF4A1, FOS, FOSB, HBEGF, HNRNPUL1, IER2, JUN, JUNB, KLF2, NR4A1, NR4A2, PTGS2, RGS1, RTN4, SLC25A25, SOCS3 and ZFP36 $(p<0.05)$.

Cluster Analyses. The upregulated genes between hypospadias samples and normal controls were selected. Cluster analyses revealed several patterns of genes and included a number of transcription factors, signal pathways, cell cycle, metabolism, nuclear receptor family and structure proteins as well as growth factor receptors (Table 1). 


\section{DISCUSSION}

Hypospadias has multifactorial origins that involve the actions of environmental factors with a genetic background [8]. The previous microarray studies indicated that, activating transcription factor 3 (ATF3), connective tissue growth factor $(C T G F)$ and cysteine-rich, angiogenic inducer 61 (CYR61) genes were upregulated in hypospadias and all three genes were also estrogen-responsive [8-10].

The ATF3 gene is upregulated in the skin of patients with hypospadias compared to normal prepuce. Also, ATF3 expression at the mRNA level in fetal mouse tissues demonstrated that its mRNA is expressed significantly more in genital tubercles from fetal mice exposed in utero to estrogens than in those of unexposed fetal mice $[4,10]$. This gene has a role in suppression of cell cycling; therefore, it had been hypothesized that its role in hypospadias might be inhibition of cell growth in urethral formation. ATF3 is upregulated in human and mouse hypospadiac tissues compared with control tissues, at both the mRNA and protein levels [8]. It has been suggested that ATF 3 may play a role in development of hypospadias as a result of exposure to estrogenic compounds [11]. Sequence variants of the $A T F 3$ gene may be involved in the genetic risk for hypospadias [12]. These genomic variants of $A T F 3$ have been reported to be present in $10.0 \%$ of patients with hypospadias [13]. In our study, we detected an upregulation of the $A T F 3$ gene by 13-fold in hypospadias tissues with respect to the controls.

The other genes that have been identified from a human microarray analysis study were $C T G F$ and CYR61. These genes were both members of the cyclin gene family and might have roles in matrix remodeling through the activation of metalloproteinases $[8,10]$. Our study only revealed an upregulation of the CYR61 gene by 5.8-6.0-fold.

Among the other 22 upregulated genes, several patterns of genes including apoptosis (FOS), apoptosis and signalling (NR4A1), metabolism (PTGS2), protein binding (RTN4), receptor activity (CD69), signalling (DUSP1, SOCS3, NR4A2, EGR1, RGS1, $H B E G F, C D 9$ ), transcription (FOSB, JUN, JUNB, IER2, ZFP36, KLF2, BTG2, HNRNPUL1), translation (EIF4A1) and transporter activites (SLC25A25) were also assessed (Table 1).
With regard to the top upregulated genes, FOS and NR4A1, were shown to induce apoptosis (Table 1). Such expression of the FOS gene has been associated with apoptotic cell death, whereas the NR4A1 gene has also been reported to induce apoptosis $[14,15]$. These two apoptotic genes (FOS, $N R 4 A 1$ ) have not been reported before.

It has been reported that apoptosis may induce external genitalia defects in fetal mouse [16]. The events leading to hypospadias formation had also been demonstrated to be associated with apoptotic and proliferative events in dorsal urethral epithelia and sinus cord [17]. However, Baskin et al. [18] indicated that hypospadias resulted from an arrest in urethral seam formation or seam remodeling but not by an epithelial apoptosis. Thus, the apoptotic genes need to be studied in a larger population.

In this study, we found a relation between hypospadias and the previously reported ATF3 and CYR61 genes. We also detected an upregulation of 22 genes in hypospadias patients that have not been reported before. Further studies including GWAS with expression studies in a larger patient group will help us to identify the candidate gene(s) in the etiology of hypospadias.

Declaration of Interest: This study was sponsored by the Scientific Research Foundation of Gazi University (01/2012-59). The authors report no conflicts of interest. The authors alone are responsible for the content and writing of this article.

\section{REFERENCES}

1. van der Zanden LF, van Rooij IA, Feitz WF, Vermeulen SH, Kiemeney LA, Knowers NV, et al. Genetics of hypospadias: are single-nucleotide polymorphisms in SRD5A2, ESR1, ESR2, ATF3 really associated with the malformation? J Clin Endocrinol Metab. 2010; 95(5): 2384-2390.

2. Putsch B, Albers N, Ludwig M. Genetic and molecular aspects of hypospadias. Eur J Pediatr Surg. 2004; 14(5): 297-302.

3. Paulozzi LJ. International trends in rates of hypo-spadias and cryptorchidism. Environ Health Perspect. 1999; 107(4): 297-302.

4. Liu B, Wang Z, Lin G, Agras K, Embers M, Wil-lingham E, et al. Activating transcription 
factor 3 is up-regulated in patients with hypospadias. Pediatr Res. 2005; 58(6): 1280-1283.

5. Kalfa N, Philibert P, Baskin LS, Sultan C. Hypo-spadias: interactions between environment and genetics. Mol Cell Endocrinol. 2011; 335(2): 89-95.

6. Li M, Qiu L, Lin T, He D, Hua Y, Yuan X, et al. c-Jun N-terminal kinase is upregulated in patients with hypospadias. Urology. 2013; 81(1): 178-183.

7. Choudhry S, Deshpande A, Qiao L, Beckman K, Sen S, Baskin LS. Genome-wide DNA methylation profiling of $\mathrm{CpG}$ islands in hypospadias. J Urol. 2012; 188(4 Suppl): 1450-1455.

8. Willingham E, Baskin LS. Candidate genes and their response to environmental agents in the etiology of hypospadias. Nat Clin Pract Urol. 2007; 4(5): 270-279.

9. Gurbuz C, Demir S, Zemheri E, Canat L, Colic $\mathrm{M}$, Caskurlu T. Is activating transcription factor 3 up-regulated in patients with hypospadias? Korean J Urol. 2010; 51(8): 561-564.

10. Wang Z, Liu BC, Lin GT, Ln CS, Lue TF, Willingham $\mathrm{E}$, et al. Up-regulation of estrogen responsive genes in hypospadias: microarray analysis. J Urol. 2007; 177(5): 1939-1946.

11. Liu B, Lin G, Willingham E, Ning H, Lin CS, Lue TF, et al. Estradiol upregulates activating transcription factor 3, a candidate gene in the etiology of hypospadias. Pediatr Dev Pathol. 2007; 10(6): 446-454.
12. Beleza-Meireles A, Töhönen V, Söderhäll C, Schwentner C, Radmayr C, Kockum I, et al. Activating transcription factor 3: a hormone responsive gene in the etiology of hypospadias. Eur J Endocrinol. 2008; 158(5): 729-739.

13. Kalfa N, Liu B, Klein O, Wang MH, Cao M, Baskin LS. Genomic variants of ATF3 in patients with hypospadias. J Urol. 2008; 180(5): 2183-2188.

14. Bakin AV, Curran T. Role of DNA 5-methylcyto-sine transferase in cell transformation by fos. Science. 1999; 283(5400): 387-390.

15. Li H, Kolluri SK, Gu J, Dawson MI, Cao X, Hobbs PD, et al. Cytochrome c release and apoptosis induced by mitochondrial targeting of orphan receptor TR3 nuclear. Science. 2000; 289(5482): 1159-1164.

16. Liu X, Zhang DY, Li YS, Zing J, He DW, Lin T, et al. Di-(2-ethylhexyl) phthalate upregulates ATF3 expression and suppresses apoptosis in mouse genital tubercle. J Occup Health. 2009; 51(1): 57-63.

17. Miyagawa S, Buchanan DL, Sato T, Ohta Y, Nishina Y, Iguchi T. Characterization of diethylstilbestrol-induced hypospadias in female mice. Anat Rec. 2002; 266(1): 43-50.

18. Baskin LS, Erol A, Jegatheesan P, Li Y, Liu W, Cunea GR. Urethral seam formation and hypospadias. Cell Tissue Res. 2001; 305(3): 379-387. 